\title{
A review of the family Protembiidae (Insecta: Eoblattida)
}

\section{Ревизия семейства Protembiidae (Insecta: Eoblattida)}

\author{
Danil S. Aristov ${ }^{1} \&$ Alexandr P. Rasnitsyn ${ }^{1,2}$ \\ А.С. Аристов, А.П. Расницын
}

\footnotetext{
1 A.A. Borisyak Paleontological Institute, Russian Academy of Science, Profsoyuznaya 123, 117997 Moscow, Russia. E-mail: danil_aristov@mail.ru; E-mail: rasna36@yahoo.com.

1 Палеонтологический институт им. А.А. Борисяка РАН, Профсоюзная 123, 117997 Москва, Россия.

${ }^{2}$ Natural History Museum, London S W7, 5 BD, England.
}

KEY WORDS: Eoblattida, Protembiidae, Telactinopterigidae, Sylvardembiidae, Permian, new taxa.

КЛЮЧЕВЫЕ СЛОВА: Eoblattida, Protembiidae, Telactinopterigidae, Sylvardembiidae, пермь, новые таксоны.

ABSTRACT. Revision of the type material of Protembiidae results in restoring of the family Telacinopterigidae and synonymising of Sylvardembiidae under Protembiidae. Telacinopterigidae are found to belong to the order Grylloblattida, and Protembiidae to the order Eoblattida. Described in Protembiidae are Repka stramenis Aristov et Rasnitsyn, gen. et sp. n., $R$. repens Aristov et Rasnitsyn, gen. et sp. n. and $R$. curta Aristov et Rasnitsyn, gen. et sp. n. from the Lower Kazanian locality Soyana in Arkhangelsk Region of Russia.

РЕЗЮМЕ. Переизучение типового материала семейства Protembiidae позволило восстановить из синонимов семейство Telactinopterigidae и свести в синонимы к Protembiidae семейство Sylvardembiidae. Telactinopterigidae отнесено к отряду Grylloblattidae, Protembiidae перенесено из гриллоблаттидовых в Eoblattida. Описаны новые протембииды: Repka stramenis Aristov et Rasnitsyn, gen. et sp. n., $R$. repens Aristov et Rasnitsyn, gen. et sp. п. и R. curta Aristov et Rasnitsyn, gen. et sp. n. из нижнеказанского местонахождения Сояна (Архангельская обл. России).

\section{Introduction}

Protembiidae is originally described as a family included only Protembia permiana Tillyard, 1937 in the order Embioptera from the Lower Permian locality Elmo in Kansas, USA [Tillyard, 1937a]. Succeeding authors referred the family to as Protorthoptera [Carpenter, 1950; 1992] or Grylloblattida [Rasnitsyn, 1980; Storozhenko, 1997; 2002]. Carpenter [1950] examined type material of Telactinopterix striatipennis Tillyard, 1937 [Tillyard, 1937b] from the same locality (Figs 12) which is the type of the family Telacinopterigidae [Carpenter, 1943], and synonymized respective two species, genera, and families. Carpenter provided a reconstruction of $P$. permiana (Fig. 2) and consider it as a member of Protorthoptera. Our revision of the type material revealed that Carpenter's reconstruction is based on the type of $T$. striatipennis and reflects morphology of that species which is distinct from that of $P$. permiana. The latter has the head with rather small eyes, pronotum is narrow trapezoid and lacking paranota, and all legs short (see below for details). Unlike this, T. striatipennis shows large eyes, short transverse pronotum with narrow, circular paranota, and mid and hind legs not shortened. These observations indicate that the fossils in question are distinct as much as at the ordinal level. The family Telactinopterigidae deserves restoration and attribution to the order Grylloblattida where it is closest to Camptoneuritidae [Aristov et al., 2010].

The family Protembiidae is considered here in a wider sense than earlier. Protembia is characteristic of big head with rather small eyes, long trapezoid pronotum lacking paranota, and short legs and ovipositor. Similar in these respect is the family Sylvardembiidae from the Lower and Middle Permian of Russia. Differences of Protembia from Sylvardembia Novokshonov, 2000 do not look justifying separation of the families (see below for details). This inference results in synonymy of the two families and transfer of the genera Sylvardembia Novokshonov, 1997, Barmaleus Novokshonov, 1997, Parbarmaleus Novokshonov, 1997, and Paratillyardembia Aristov, 2000 into Protembiidae. Of them, Sylvardembia and Barmaleus were described in Tillyardembiidae [Novokshonov, 1997a] and later established as a separate family in the order Grylloblattida [Novokshonov, 2000]. Revision of the type and additional material of Sylvardembiidae revealed their SC as meeting R rather than $\mathrm{C}$. This character taken together with general similarity of their body structure to that of Tillyardembiidae implies position of Sylvardembiidae in the order Eoblattida where Tillyardembiidae have been transferred to recently [Aristov \& Rasnitsyn, 2009]. In that order, Protembiidae along with Tillyardembiidae look like a dwarf relatives of the characteristic Carbonifer- 


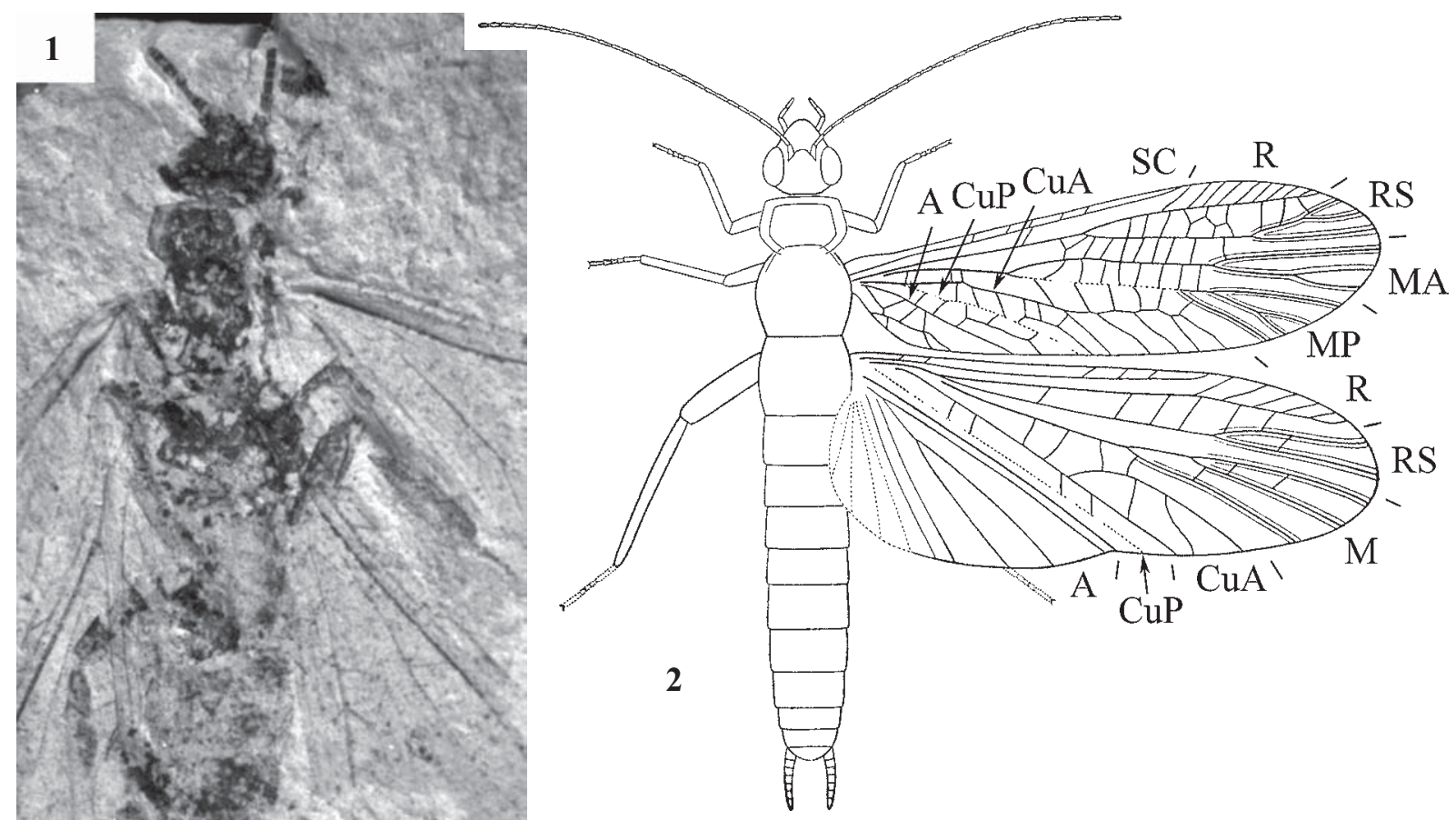

Figs 1-2. Telactinopterix striatipennis Tillyard, 1937 (Grylloblattida, Telacinopterigidae): 1 - paratype PMYU, No. 15559 (original photograph by Una Farrell (PMYU)), 2 - reconstruction (modified after Carpenter, 1950); Elmo, Kansas, USA, Leonardian, Lower Permian. Unless stated otherwise, scale bar is $1 \mathrm{~mm}$.

Puc. 1-2. Telactinopterix striatipennis Tillyard, 1937 (Grylloblattida, Telacinopterigidae): 1 — паратип PMYU, No. 15559 (оригинальная фотография Уны Фаррелл (PMYU)), 2 - реконструкция (по Carpenter, 1950 с изменениями); Эльмо, Канзасс, США, леонардский ярус, нижняя пермь. Длина масштабной линейки соответствует 1 мм, если не указано иное.

ous family Spanioderidae, as it was interpreted by Béthoux [2008] (Fig. 3).

Sylvardembia pectinata Novokshonov, 2000 represents a special case because its forewing reveals SC meeting $\mathrm{C}$ and not $\mathrm{R}$. The species was described based on a detached forewing [Novokshonov, 2000]. A more complete fossil was found recently (Fig. 4) which differs significantly, additionally, from all Protembiidae in having wide paranota and apparently 5-segmented tarsus, as opposed to 3-segmented tarsus of Protembiidae. Wing and body structure of "Sylvardembia" pectinata does not contradict its attribution to Grylloblattida. Taxonomic position of that species deserves a separate publication to be considered.

\section{Order Eoblattida Handlirsch, 1906}

Family Protembiidae Tillyard, 1937

Protembiidae: Tillyard, 1937a: 243; Carpenter, 1950: 207; Sharov, 1962: 124; Rasnitsyn, 1980: 152; Carpenter, 1992: 115; Storozhenko, 1997: 7; 1998: 91; 2002: 279.

Sylvardembiidae: Novokshonov, 2000: 44; Storozhenko, 2002: 297; Aristov, 2004: 85, syn. n.

TYPE GENUS. Protembia Tillyard, 1937.

DIAGNOSIS. Size small (forewing length $6.1-11.0 \mathrm{~mm}$ ). Head big, triangular. Pronotum lacking paranota. Legs short, subequal in length, mid pair shifted posterior, tarsus trimerous, ovipositor short. SC meeting R, RS, M, and CuA anastomos- ing since before forewing midlength, forming large backward comb running close to $\mathrm{R}$. CuA branching from near its base.

COMPOSITION. Six genera and nine species, as follows: Protembia pemiana from Elmo (Leonardian of Kansas, USA); Sylvardembia with two species S. tamaena Novokshonov, 1997 and S. matura Aristov, 2000, Barmaleus dentatus Novokshonov, 1997, and Paratillyardembia sepicolorata Aristov, 2000 from Kungurian of Tshekarda (Perm Province, Russia), Parbarmaleus sojanensis Novokshonov, 1997, and Repka Aristov et Rasnitsyn, gen.n. with $R$. stramentum Aristov et Rasnitsyn sp.n., $R$. repens Aristov et Rasnitsyn sp.n. and R. curta Aristov et Rasnitsyn sp. n. from Lower Kazanian of Soyana (Arkhangelsk Reg., Russia).

COMPARISON. Similar to Spanioderidae in general appearance and, venationally, in anastomosis of $\mathrm{M}+\mathrm{CuA}$ and backward comb of CuA. Differs from Spanioderidae in size smaller, head bigger, legs short and with tarsus 3-segmented, and venationally, in anastomosis of $\mathrm{M}+\mathrm{CuA}$ longer, and in having extensive backward comb of $\mathrm{CuA}(+\mathrm{RS},+\mathrm{M})$. Similar in size and general appearance also to Tillyardembiidae, but unlike the latter the tarsus is trimerous, ovipositor short, and $\mathrm{CuA}$ branching early and forming anastomosis with $\mathrm{M}$.

Genus Protembia Tillyard, 1937

Protembia: Tillyard, 1937a: 243; Carpenter, 1950: 208; 1992 : 117; Zalessky, 1950: 49; Storozhenko, 1997: 7; 1998: 91.

TYPE SPECIES. P. permiana Tillyard, 1937.

COMPOSITION. Type species from Leonardian of Elmo in Kansas, USA. 


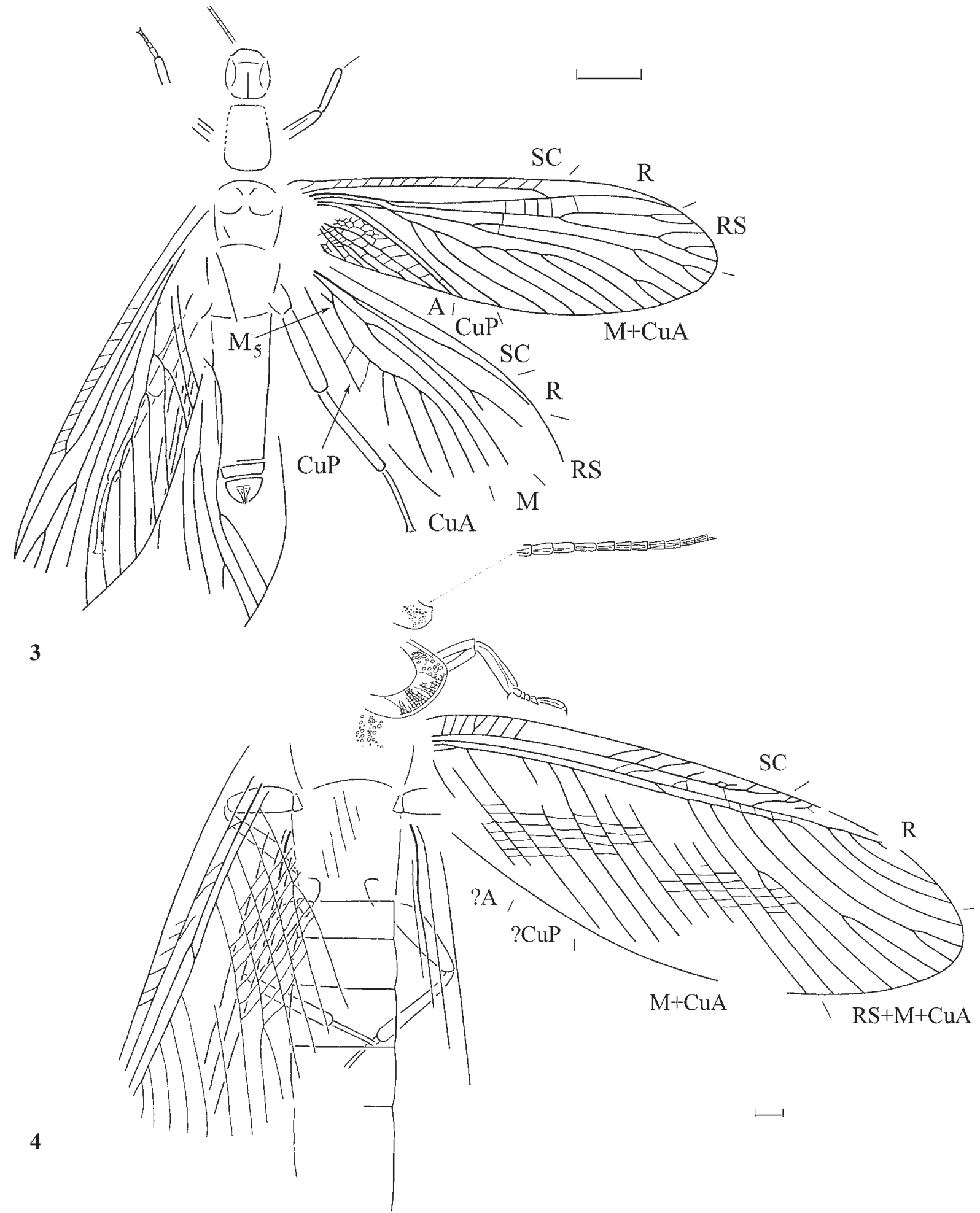

Fig. 3. Miamia bronsoni Dana, 1864 (Eoblattida, Spanioderidae), reconstruction based on spec. USNM 38817 and FMNH PE 30369 (orig.); Mazon Creek, Illinois, USA; Desmoinesian Stage, Upper Carboniferous. Scale bar is $5 \mathrm{~mm}$.

Рис. 3. Miamia bronsoni Dana, 1864 (Eoblattida, Spanioderidae), реконструкция на основе экз. USNM 38817 и FMNH PE 30369 (ориг.); Мэзон Крик, Иллинойс, США; десмоинский ярус, верхний карбон. Длина масштабной линейки соответствует 5 мм.

Fig. 4. "Sylvardembia" pectinata (Novokshonov, 2000) (Grylloblattida), reconstruction based on holotype PIN, no. 1700/1012 and spec. PIN, no. 1700/1080 (orig.); Chekarda, Perm Region, Russia; Kungurian, Lower Permian.

Рис. 4. "Sylvardembia" pectinata (Novokshonov, 2000) (Grylloblattida), реконструкция на основе голотипа ПИН, № 1700/1012 и экз. ПИН, №. 1700/1080 (ориг.); Чекарда, Пермский край, кунгурский ярус, нижняя пермь. 

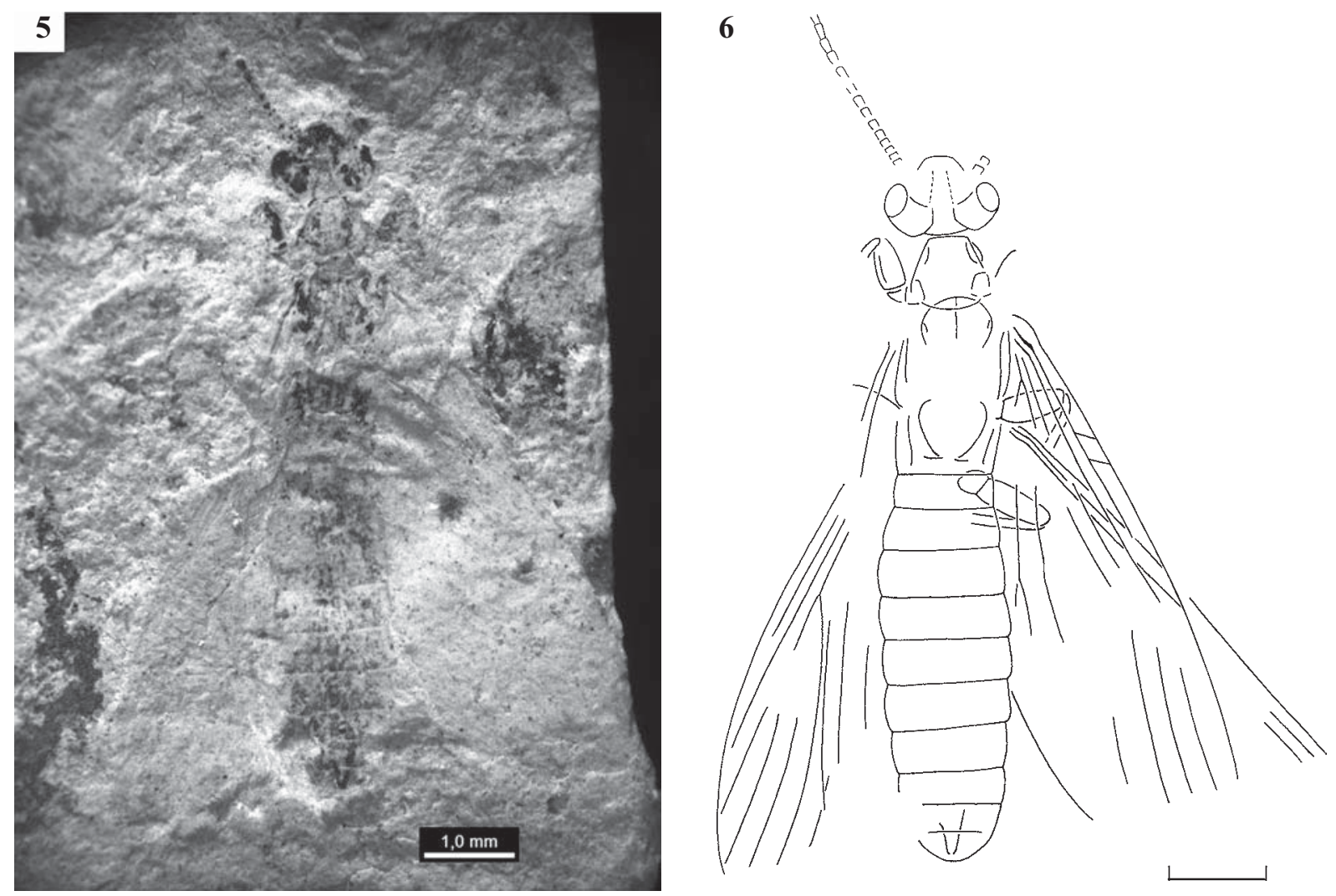

Figs 5-6. Protembia permiana Tillyard, 1937, holotype PMYU, No. 15507: 5 - general view (original by Una Farrell (PMYU)), 2 interpretation.

Pис. 5-6. Protembia permiana Tillyard, 1937, голотип PMYU, o. 15507: 5 - общий вид (оригинальная фотография Уны Фаррелл (PMYU)), 6 - интерпретация.

Protembia permiana Tillyard, 1937

Figs 5-6

Protembia permiana: Tillyard, 1937a: 245, figs 1-3; Zalessky, 1950: 49, fig. 13; Carpenter, 1950: 208, fig. 11; 1992: 117, fig. 68 , 2; Storozhenko, 1997: 7, fig. 6; 1998: 91, fig. 163

HOLOTYPE. PMYU, No. 15507; imprint of entire insect; Elmo locality, $5 \mathrm{~km}$ SE of Elmo town, Banner Township, southern Dickinson County, north-central Kansas, USA; Carlton Mb., Wellington Fm., Sumner Group, Lower Leonardian Stage, Lower Permian; kept in Peabody Museum of Natural History, Yale University, New-Heven, USA.

Genus Sylvardembia Novokshonov, 1997

Sylvardembia: Novokshonov, 1997a: 41; 2000: 44; Aristov, 2000: 48; 2004: 85; Aristov \& Rasnitsyn, 2009: 257.

TYPE SPECIES. S. tamaena Novokshonov, 1997.

COMPOSITION. Two species from Kungurian Stage, Lower Permian (Russia).

\section{Sylvardembia tamaena Novokshonov, 1997}

Figs 7-8

Sylvardembia tamaena: Novokshonov, 1997a: 42, fig. 2; Tabl. V, fig.2; Aristov, 2000: 48; 2004: 85.

HOLOTYPE. PIN, no. 4987/104; imprint of entire insect; Chekarda locality, left bank of Sylva River near mouth of Chekarda River, Suksun District, Perm Province, European Russia; Koshelevka Formation, Iren' Horizon, Kungurian Stage, Lower Permian; Novokshonov V.G. leg. 1987-1999; kept in Paleontological Institute, Russian Academy of Science, Moscow.

\section{Sylvardembia matura Aristov, 2000}

Fig. 9

Sylvardembia matura: Aristov, 2000: 48, figs 1, 3a; 2004 : 85.

HOLOTYPE. PIN, no. 1700/1935; imprint of entire insect; Chekarda locality, left bank of Sylva River near mouth of Chekarda River, Suksun District, Perm Province, European Russia; Koshelevka Formation, Iren' Horizon, Kungurian Stage, Lower Permian; Sharov A.G. leg. 19591961; kept in Paleontological Institute, Russian Academy of Science, Moscow.

\section{Genus Barmaleus Novokshonov, 1997}

Barmaleus: Novokshonov, 1997a: 42; 1997b: 203; 2000: 44; Aristov, 2004: 85; Aristov \& Rasnitsyn, 2009: 257.

TYPE SPECIES. B. dentatus Novokshonov, 1997.

COMPOSITION. Type species only.

\section{Barmaleus dentatus Novokshonov, 1997}

Fig. 10

Barmaleus dentatus: Novokshonov, 1997a: 43, fig. 3, tabl. V, fig. 3, 4; 1997b: 203, fig. 1; Aristov, 2004: 85 .

HOLOTYPE. PIN, no. 4987/105; imprint of entire insect; Chekarda locality, left bank of Sylva River near mouth of Chekarda River, Suksun District, Perm Province, European Russia; Koshelevka Formation, Iren' Horizon, Kungurian Stage, Lower Permian; Novokshonov V.G. leg. 19871999; kept in Paleontological Institute, Russian Academy of Science, Moscow. 

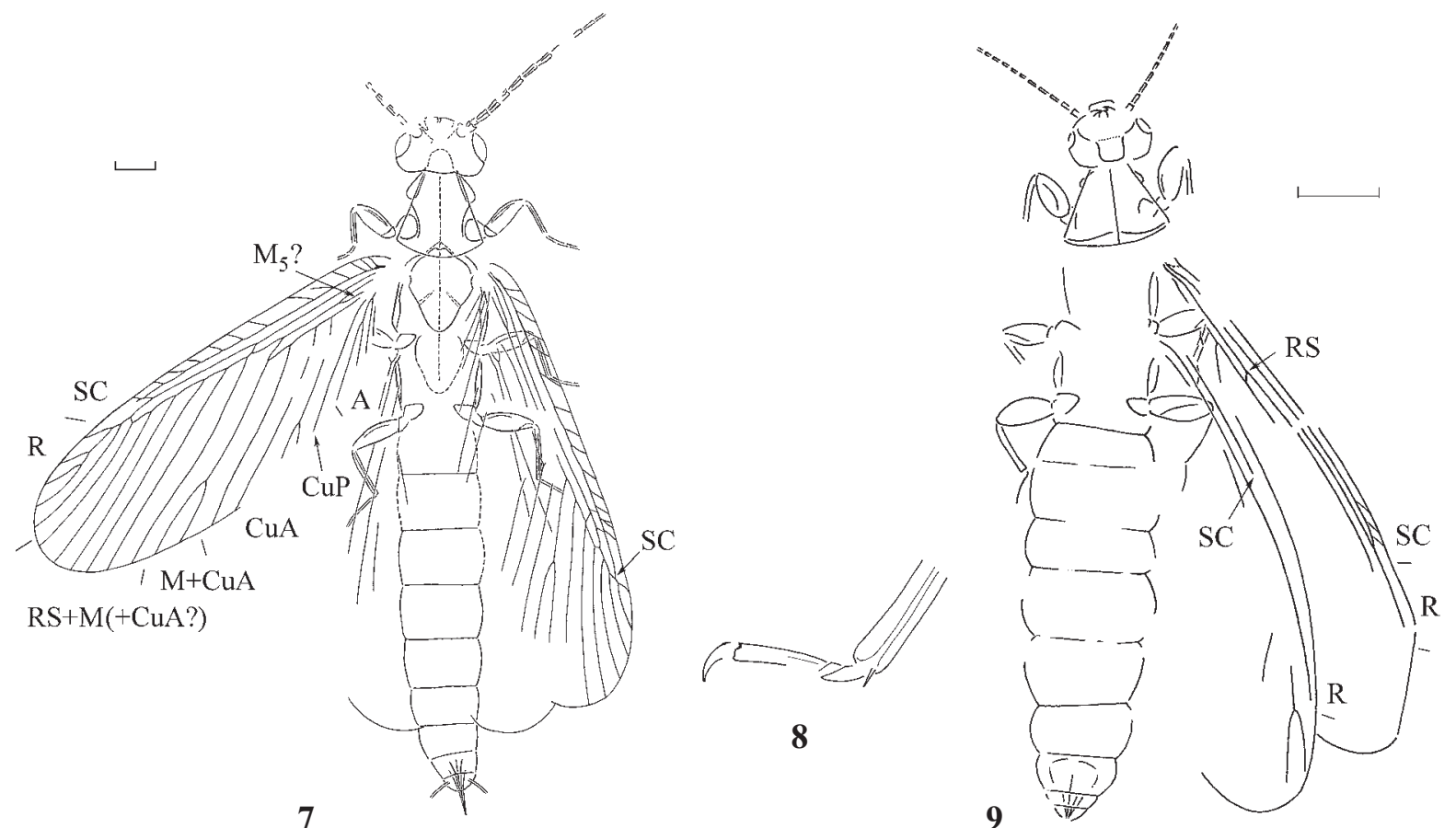

Figs 7-9. Members of genus Sylvardembia: 7-8 - S. tamaena: 7 - reconstruction based on holotype PIN, no. 4987/104, general appearance, 8 - hind tarsus (orig.); $9-$ S. matura, holotype PIN, no. 1700/1935, general appearance (modified after Aristov, 2000); Chekarda, Perm Region, Russia; Kungurian, Lower Permian.

Рис. 7-9. Представители рода Sylvardembia : 7-8 - S. tamaena: 7 - реконструкция на основе голотипа ПиН, № 4987/104, общий вид, 8 - задняя лапка (ориг.); 9 - S. matura, голотип ПИН, № 1700/1935, общий вид (по Aristov, 2000 с изменениями); Чекарда, Пермский край, кунгурский ярус, нижняя пермь.
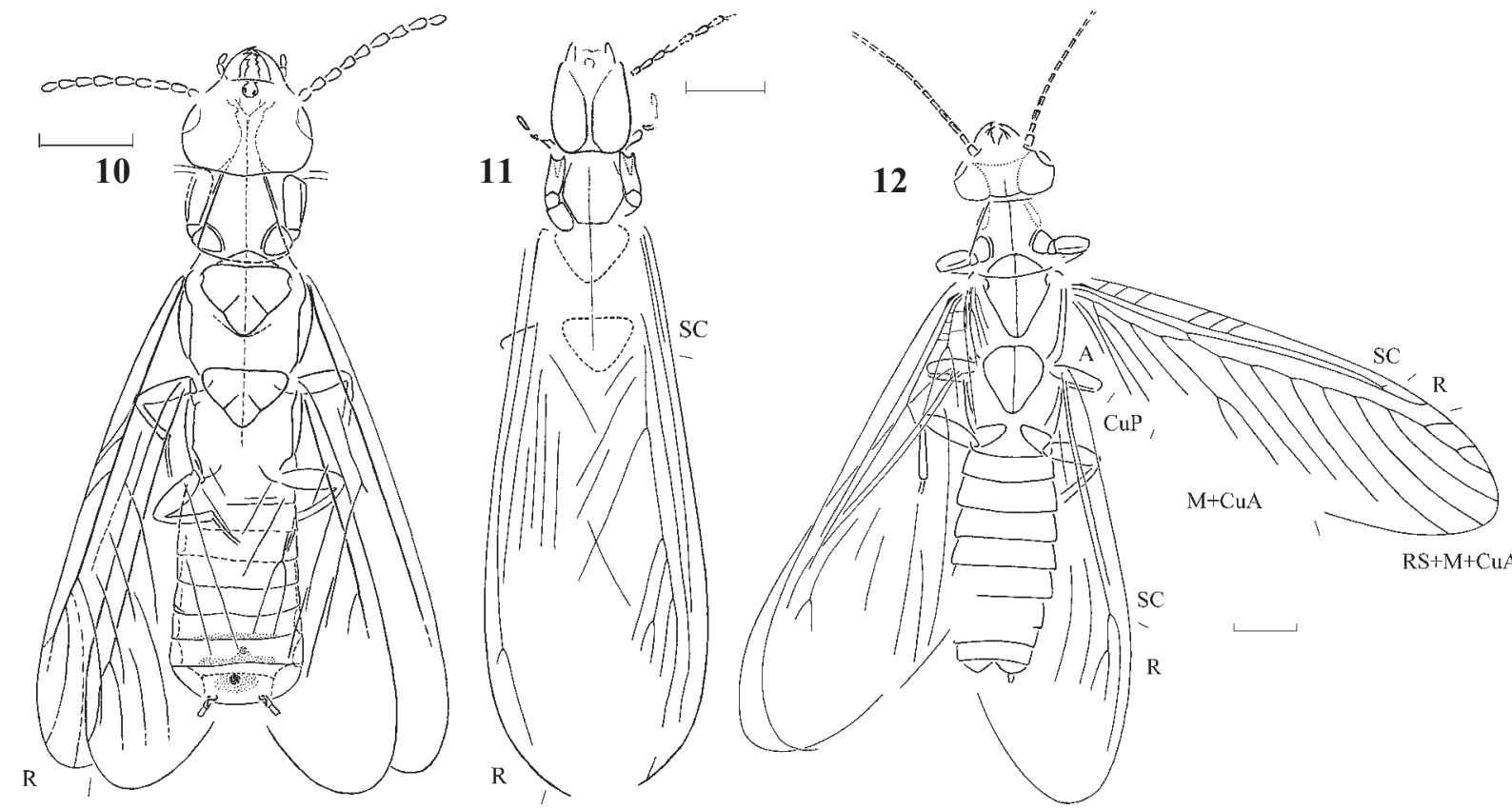

Figs 10-12. Members of family Protembiidae: 10 - Barmaleus dentatus, holotype PIN, No. 4987/105, general appearance (from Novokshonov, 1997a); 11 - Parbarmaleus sojaensis, holotype PIN, No.3353/339, general appearance (modified after Novokshonov, 1997b); 12 - Paratillyardembia sepicolorata, reconstruction based on holotype PIN, No. 1700/1179, general appearance (orig.); 10, 12 Chekarda, Perm Region, Russia; Kungurian, Lower Permian; 11 - Soyana, Arkhangelsk Region, Russia; Lower Kazanian, Middle Permian.

Рис. 10-12. Представители семейства Protembiidae: 10 - Barmaleus dentatus, голотип ПИН, № 4987/105, общий вид (из Novokshonov, 1997a); 11 — Parbarmaleus sojaensis, голотип ПИН, № 3353/339, общий вид (по Novokshonov, 1997b, с изменениями); 12 - Paratillyardembia sepicolorata, реконструкция на основе голотипа ПИН, № 1700/1179, общий вид (ориг.); 10, 12 - Чекарда, Пермский край, кунгурский ярус, нижняя пермь; 11 - Сояна, Архангельская обл., Россия; нижнеказанский подъярус, средняя пермь. 


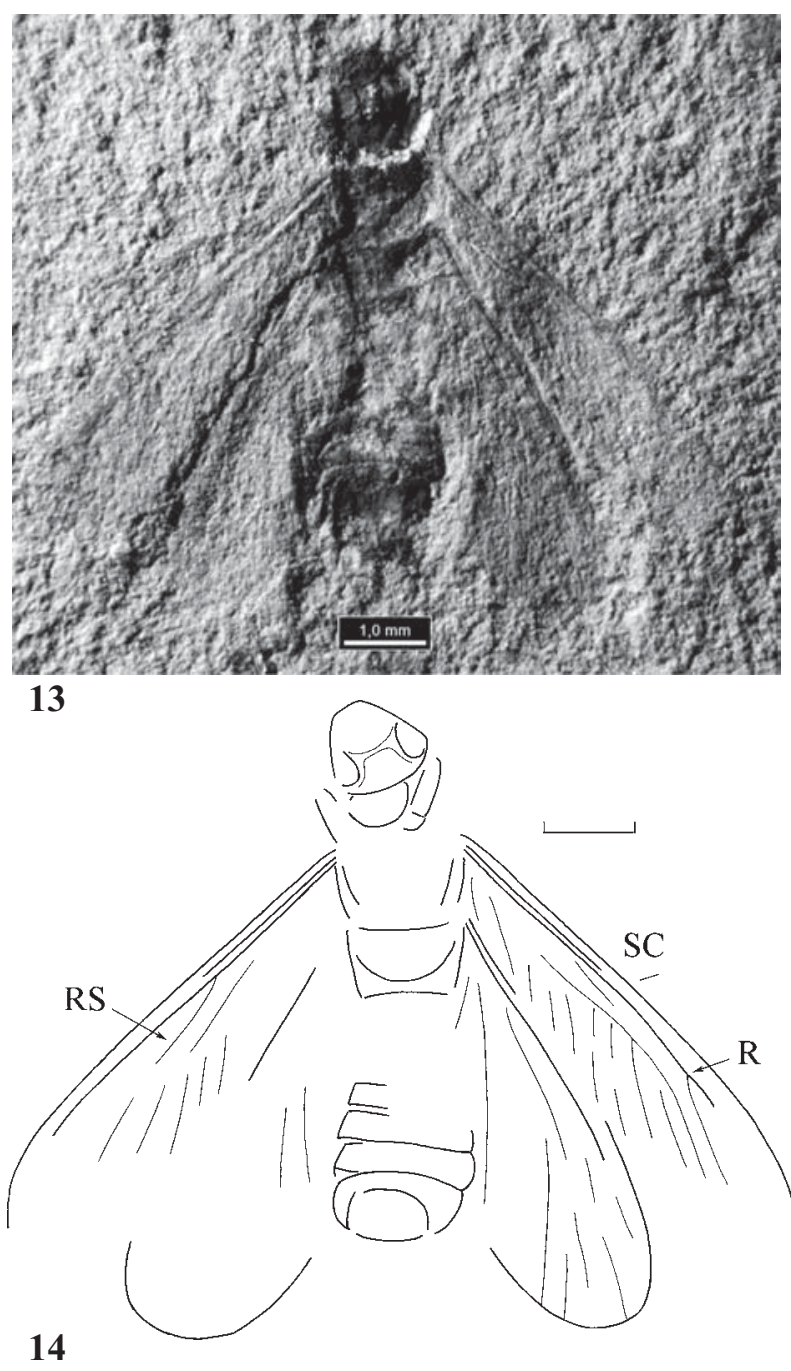

Figs 13-14. Repka stramenis Aristov et Rasnitsyn, sp.n., holotype PIN, no. 3353/539, general appearance; Soyana, Arkhangelsk Region, Russia; Lower Kazanian, Middle Permian.

Рис. 13-14. Repka stramenis Aristov et Rasnitsyn, sp.n., голотип ПИН, №. 3353/539, общий вид; Сояна, Архангельская обл., Россия; нижнеказанский подъярус, средняя пермь.

Genus Parbarmaleus Novokshonov, 1997, stat. nov.

Parbarmaleus: Novokshonov, 1997b: 203.

TYPE SPECIES. P. sojaensis Novokshonov, 1997.

COMPOSITION. Type species only.

REMARKS. Parbarmaleus was described as a subgenus of Barmaleus (Novokshonov, 1997b). However, differences in the body structure look justifying generic level distinction of the taxa, the more so that the subgenus rank is not generally used in the eoblattid taxonomy.

\section{Parbarmaleus sojaensis Novokshonov, 1997}

\section{Fig. 11}

Parbarmaleus sojaensis: Novokshonov, 1997b: 203, fig. 1. HOLOTYPE. PIN, no. 3353/339; imprint of entire insect; Soyana locality, right bank of Soyana River 56-60 km from mouth, Mezen' District, Arkhangelsk Region, Russia; Iva-Gora Beds, Lower Kazanian Substage, Kazanian Stage, Middle Permian; Sharov A.G. leg. 1972; kept in Paleontological Institute, Russian Academy of Science, Moscow.
Genus Paratillyardembia Aristov, 2000

Paratillyardembia: Aristov, 2000: 48; 2004: 85. TYPE SPECIES. P. sepicolorata Aristov, 2000. COMPOSITION. Type species only.

Paratillyardembia sepicolorata Aristov, 2000 Fig. 12

Paratillyardembia sepicolorata: Aristov, 2000: 49, fig. 2; 2004: 85.

HOLOTYPE. PIN, no. 1700/1179; imprint of entire insect; Chekarda locality, left bank of Sylva River near mouth of Chekarda River, Suksun District, Perm Province, European Russia; Koshelevka Formation, Iren' Horizon, Kungurian Stage, Lower Permian; Sharov A.G. leg. 19591961; kept in Paleontological Institute, Russian Academy of Science, Moscow.

Genus Repka Aristov et Rasnitsyn gen. n.

TYPE SPECIES. R. stramenis Aristov et Rasnitsyn, sp. $\mathbf{n}$.

DIAGNOSIS. Small insects. Head with large eyes and short antennae (shorther than head). Pronotum shorter and narrower than head, narrowing backward. Fore legs short, not distinctly modified. Meso- and metanotum transverse. Forewing with fore margin straight, costal space wider than subcostal one in basal third, SC reaching beyond wing midlength, $\mathrm{R}$ with hind branches. Wings extending beyond abdomen apex.

COMPOSITION. Three species from the Middle Permian of northern European Russia.

ETIMOLOGY. Arbitrary composition of letters. Gender feminine.

\section{Repka stramenis Aristov et Rasnitsyn, sp. n.} Figs 13-14

MATERIAL. Holotype PIN, no. 3353/539; imprint of entire insect; Soyana locality, right bank of Soyana River 56-60 km from mouth, Mezen' District, Arkhangelsk Region, Russia; Iva-Gora Beds, Lower Kazanian Substage, Kazanian Stage, Middle Permian; Sharov A.G. leg. 1972; kept in Paleontological Institute, Russian Academy of Science, Moscow.

DESCRIPTION. Head slightly shorter than wide, with eyes placed basal. Pronotum roundish trapezoid, half as long and two third as wide as head. Fore femur longer than pronotum, 3.6 times as long as wide. Mesonotum longer than metanotum, metanotum transverse. Forewing with large posterior comb of oblique veins, RS starting before wing midlength, free for some distance, then fusing with $\mathrm{M}$.

ETIMOLOGY. After stramen, the Latin for (leaf) litter.

\section{Repka repens Aristov et Rasnitsyn, sp. n.}

\section{Figs $15-17$}

MATERIAL. Holotype PIN no. 3353/307; imprint of entire insect; Soyana locality, right bank of Soyana River 56-60 km from mouth, Mezen' District, Arkhangelsk Region, Russia; Iva-Gora Beds, Lower Kazanian Substage, Kazanian Stage, Middle Permian; Sharov A.G. leg. 1972; kept in Paleontological Institute, Russian Academy of Science, Moscow.

DESCRIPTION. Head as long as wide, with eyes placed near its midlength. Antenna widening toward apex, with articles as long as wide. Pronotum ca. 0.6 times as wide as and 0.35 as long as head. Fore femur and trochanter combined about half as long as head wide. Metanotum about as long as wide, longer than mesonotum.

ETIMOLOGY. The name is the Latin for creeping.

\section{Repka curta Aristov et Rasnitsyn, sp. n.} Figs 18-19

MATERIAL. Holotype PIN, no. 3353/232; imprint of entire insect; Soyana locality, right bank of Soyana River 56-60 km from 


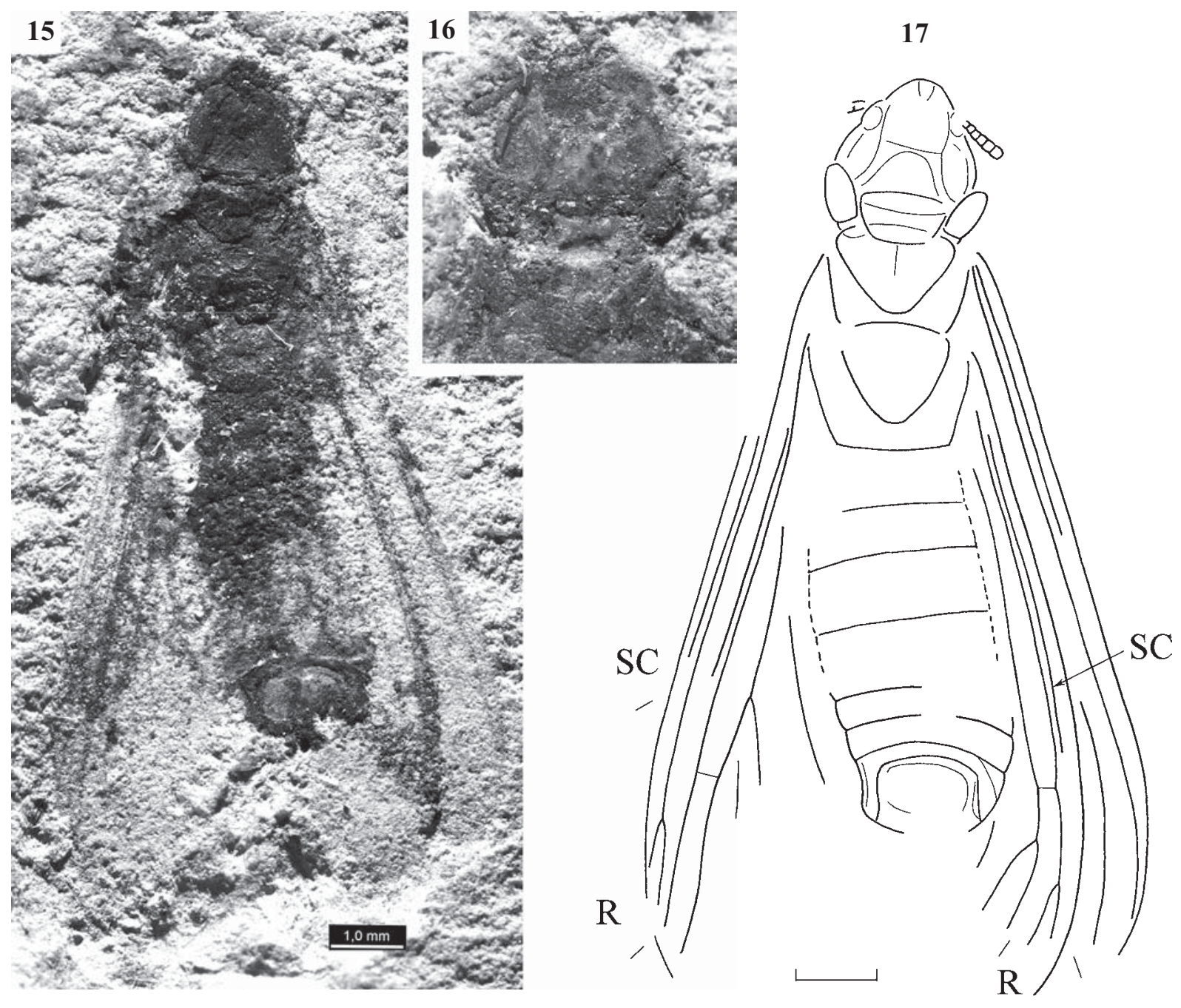

Figs 15-17. Repka repens Aristov et Rasnitsyn, sp.n., holotype PIN, no. 3353/307, 15, 17 - general appearance, 16 - head and pronotum; Soyana, Arkhangelsk Region, Russia; Lower Kazanian, Middle Permian.

Рис. 15-17. Repka repens Aristov et Rasnitsyn, sp.n., голотип ПИН, №. 3353/307, 15, 17 - общий вид, 16 — голова и пронотум; Сояна, Архангельская обл., Россия; нижнеказанский подъярус, средняя пермь.

mouth, Mezen' District, Arkhangelsk Region, Russia; Iva-Gora Beds, Lower Kazanian Substage, Kazanian Stage, Middle Permian; Sharov A.G. leg. 1972; kept in Paleontological Institute, Russian Academy of Science, Moscow.

DESCRIPTION. Head as long as wide. Fore femur 1.4 times as long as wide. Mesonotum triangular, as long as wide, metanotum trapezoid, transverse. SC ending in almost symmetric fork well beyond forewing midlength. $\mathrm{R}$ with posterior branch near hind wing apical quarter. femur).

ETIMOLOGY. The Latin for short (after form of the fore

\section{Key to genera And species of Protembitdae}

1 (12) Pronotum not transverse, usually trapezoid, narrowing forward, not shorter or slightly shorter than head.

2 (3) RS branches almost parallel to wing axis in distal wing third. Head transverse, much narrowed behind eyes. Length of body $7.3 \mathrm{~mm}$, forewing $6.2 \mathrm{~mm}$, hind wing $5 \mathrm{~mm}$...

$$
\text { Protembia permiana Tillyard, } 1937 \text { (Figs 5-6) }
$$

3 (2) RS branches much oblique in distal wing third. Head slightly or not at all narrowed behind eyes.
4 (7) Preradial space not wider than interradial one. Head very large, prognathous. Antena thread-like (not narrowing apical). Forewing length $8 \mathrm{~mm}$ or less.

5 (6) Head transverse (excluding mandibles). Pronotum trapezoid (narrowing foreward). Length of body 7.8 , forewing 6.1, hind wing $4.5 \mathrm{~mm}$.

Barmaleus dentatus Novokshonov, 1997 (Fig. 7)

6 (5) Head 1.4 times as long as wide, pronotum long, rounded hexagonal, widest near midlength. Length of forewing 7 , hind wing $6.5 \mathrm{~mm}$

... Parbarmaleus sojaensis Novokshonov, 1997 (Fig. 8)

7 (4) Preradial space much wider than interradial one. Head not very large and not prognathous. Antenna bristle-like (narrowing apical). Forewing longer than $8 \mathrm{~mm}$

8 (11) $\mathrm{CuA}$ meeting hind branch of $\mathrm{M}$ in forewing basal quarter or third. Interradial space narrower than costal one. Fore femur and trochanter jointly half as long as pronotum wide. .......Sylvardembia Novokshonov, 1997

9 (10) Eyes of moderate size, femora not wided. Length of body 17.2 , forewing 11 , hind wing $9 \mathrm{~mm}$.

S. tamaena Novokshonov, 1997 (Figs 9-10) 

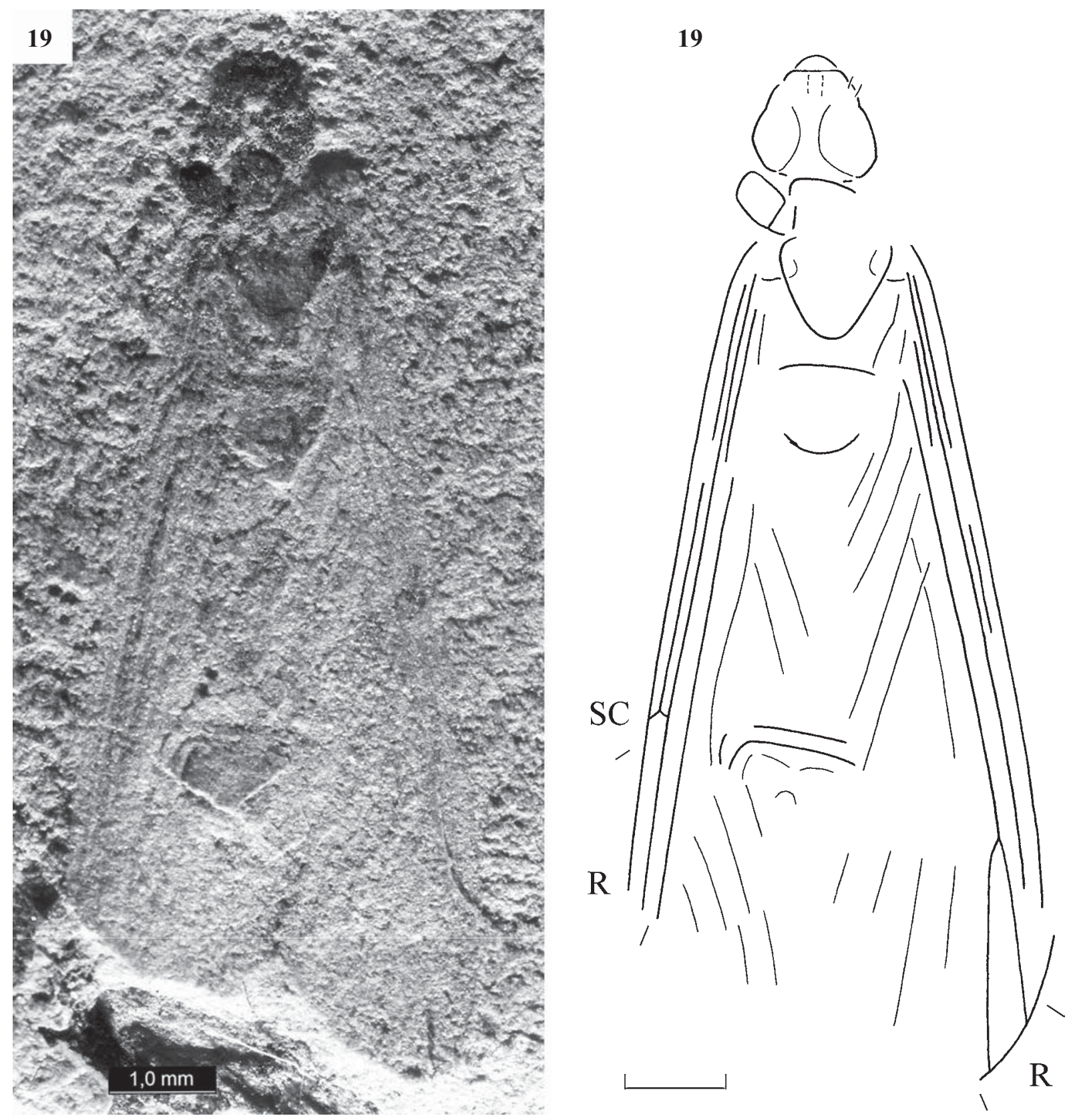

Figs 18-19. Repka curta Aristov et Rasnitsyn, sp.n., holotype PIN, no. 3353/232, general appearance; Soyana, Arkhangelsk Region, Russia; Lower Kazanian, Middle Permian.

Pис. 18-19. Repka curta Aristov et Rasnitsyn, sp.n., голотип ПИН, №. 3353/232, общий вид; Сояна, Архангельская обл., Россия; нижнеказанский подъярус, средняя пермь.

10 (9) Eyes small, femora widened. Length of body 11.5, forewing 9 , hind wing $7.5 \mathrm{~mm}$.

S. matura Aristov, 2000 (Fig. 11)

11 (8) $\mathrm{M}$ and $\mathrm{CuA}$ with common stalk. Interradial space as wide as costal one, Fore femur and trochanter jointly one third as long as pronotum wide, Length of body 8.6, forewing 8.5 , hind wing $7 \mathrm{~mm}$.

... Paratillyardembia sepicolorata Aristov, 2000 (Fig. 12)

12 (1) Pronotum much transverse, narrowing backward. Head several times as long as pronotum, rounded triangular, narrowing forward.

Repka Aristov et Rasnitsyn, gen.n.
13 (16) Fore femur at least twice as long as wide.

14 (15) Head twice as long as pronotum, fore femur longer than pronotum. Length of body 5.5 , forewing 6 , hind wing $5 \mathrm{~mm}$.

... R. stramenis Aristov et Rasnitsyn, sp.n. (Figs 13-14)

15 (14) Head 2.8 times as long as pronotum, fore femur as long as pronotum. Length of body 9.5 , forewing ca. 10, hind wing ca. $9 \mathrm{~mm}$.

....... R. repens Aristov et Rasnitsyn, sp.n. (Figs 15-17)

16 (13). Fore femur 1.4 times as long as wide. Length of body 7 , forewing ca. 9 , hind wing ca. $7.5 \mathrm{~mm}$ ....... R. curta Aristov et Rasnitsyn, sp.n. (Figs 18-19) 
ACKNOWLEDGEMENTS. The present study is supported in part by the Presidium RAS Program "Origin of the biosphere and evolution of geo-biological systems" and the Russian Foundation for Basic Research Grants \#\# 09-0401241 and 10-04-01713. Authors appreciate immense help of Dr. Tim White and Ms Una Farrell (Peabody Museum of Natural History, Yale University, New Haven, USA) who prepared photographs of the type specimens of Protembia permiana and Telacinopteryx striatipennis.

\section{References}

Aristov D.S. 2000. [New insects of the order Grylloblattida (Insecta) from the Lower Permian of the Middle Urals] // Paleontol. Zh No.5. P.48-50 [in Russian, English translation: Paleontol. J. Vol.34. No.5. P. 519-521].

Aristov D.S. 2004. The fauna of grylloblattid insects (Grylloblattida) of the Lower Permian locality of Tshekarda // Paleontol. J. Vol.38. Suppl.2. P.80-145.

Aristov D.S., Rasnitsyn A.P. 2009. The family Tillyardembiidae Zalessky, 1938 and the system of the plecopteroid insects / Russian Entomol. J. Vol.18. No.3. P. 257-264.

Aristov D.S., Storozhenko S.Yu. \& Cui Y.Y. 2010. Review of the Permian Camptoneuritidae (Insecta: Grylloblattida) // Acta Geologica Sinica. Vol.84. No.4. P.756-761.

Béthoux O. 2008. Revision and phylogenetic affinities of the Lobeattid species bronsoni Dana, 1864 and silvatica Laurentiaux \& Laurentiaux-Vieira, 1980 (Pennsylvanian; Archaeorthoptera) // Arthropod Systematics \& Phylogeny. Vol.66. No.2. P. 145-163.

Carpenter F.M. 1943. The Lower Permian insects of Kansas. Pt. 9. The orders Neuroptera, Rhaphidiodea, Caloneurodea, and Protorthoptera, with additional Protodonata and Megasecoptera // Proc. Amer. Acad. Arts and Sci. Vol.75. No.2. P.55-84.

Carpenter F.M. 1950. The Lower Permian insects of Kansas. Pt. 10 The order Protoperlaria: the family Liomopteridae and its relatives // Proc. Amer. Acad. Arts and Sci. Vol.78. No.4. P. $183-219$.
Carpenter F.M. 1992. Treatise on Invertebrate Paleontology. Vol.3: Superclass Hexapoda // Boulder and Lawrence. 655 p.

Novokshonov V.G. 1997a. [New Taxa of Fossil Insects from the Lower Permian of the Middle Urals] // Paleontol. Zh. No.4. P.39-44 [in Russian, English translation: Paleontol. J. Vol.31. No.4. P.383-388].

Novokshonov V.G. 1997b. [New Barmaley from the Upper Permian of the Apkhangelsk Region (Insecta; Grylloblattida; Tillyardembiidae)] // Vestnik Permskogo Universiteta. Ser. Biologiya. No.3. P.202-205 [in Russian].

Novokshonov V.G. 2000. [New fossil insects (Insecta: Grylloblattida, Ordinis incertis) from the Lower Permian of the Middle Urals] // Paleontol. Zh. No.5. P.42-47 [in Russian, English translation: Paleontol. J. Vol.34. No.5. P.513-518]

Rasnitsyn A.P. 1980. [Order Grylloblattida Walker, 1914] // Rodendorf B.B., Rasnitsyn A.P. (eds.). Istoricheskoe razvitie klassa nasekomykh. M.: Nauka. Tr. Paleontol. in-ta. Vol.178. P.150154 [in Russian].

Sharov A.G. 1962. [Orders Protoblattodea, Paraplecoptera] // In: Fundamentals of Paleontology: Arthropoda. Tracheata, Chelicerata. Moscow: Nauka. P.116-138 [in Russian]

Storozhenko S.Yu. 1997. Classification of order Grylloblattida (Insecta) with description of new taxa // Far East. Entomol. No.42. P.1-20.

Storozhenko S.Yu. 1998. [Systematics, phylogeny and evolution of the grylloblattids (Insecta: Grylloblattida)] // Vladivostok: Dal'nauka. 207p. [in Russian].

Storozhenko S.Yu. 2002. Order Grylloblattida Walker, 1914 // Rasnitsyn A.P., Quicke D.L.Q. (eds.). History of Insects. Dordrecht: Kluwer Acad. Publ. P.278-281.

Tillyard R.J. 1937a. Kansas Permian insects. Part 18. The order Embiaria // Amer. J. Sci. Vol.33. P.241-251.

Tillyard R.J. 1937b. Kansas Permian insects. Part 19. The order Protoperlaria (Continued). Family Probnidae // Amer. J. Sci. Vol.33. P.401-426.

Zalessky Yu.M. 1950. [Permian insects from the basin of Sylva River, and problems of evolution in the Class Insecta. IV. New representatives of the group Epiembiodea and evolution of the webspinners] // Problemy evolutsii. Vol.1. P. 41-60 [in Russian]. 